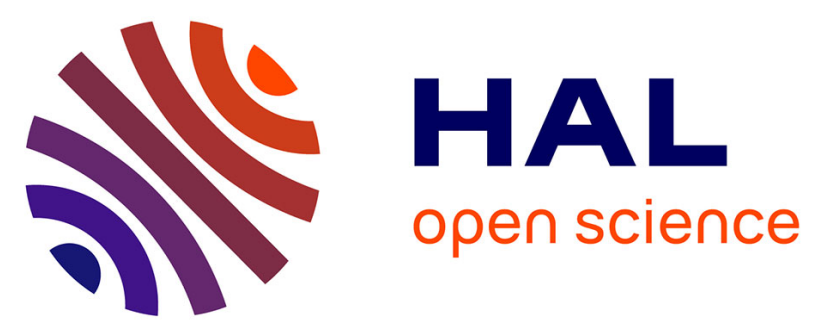

\title{
Dernière étape avant Rome? Les élections régionales italiennes du 16 avril 2000
}

\author{
Mario Caciagli, Carlo Baccetti, Emmanuel Négrier
}

\section{To cite this version:}

Mario Caciagli, Carlo Baccetti, Emmanuel Négrier. Dernière étape avant Rome? Les élections régionales italiennes du 16 avril 2000. Pôle Sud - Revue de science politique de l'Europe méridionale, 2000, Qui gouverne les villes? p, 13, pp.121-136. 10.3406/pole.2000.1091 . hal-02552459

\section{HAL Id: hal-02552459 \\ https://hal.science/hal-02552459}

Submitted on 28 Apr 2020

HAL is a multi-disciplinary open access archive for the deposit and dissemination of scientific research documents, whether they are published or not. The documents may come from teaching and research institutions in France or abroad, or from public or private research centers.
L'archive ouverte pluridisciplinaire HAL, est destinée au dépôt et à la diffusion de documents scientifiques de niveau recherche, publiés ou non, émanant des établissements d'enseignement et de recherche français ou étrangers, des laboratoires publics ou privés.

\section{(ㅇ)(1) $\$$}

Distributed under a Creative Commons Attribution - NonCommercial - NoDerivatives 44.0 


\title{
Dernière étape avant Rome ? Les élections régionales italiennes du
} 16 avril 2000

\author{
Mr Mario Caciagli, Mr Carlo Baccetti, Mr Emmanuel Négrier
}

\section{Citer ce document / Cite this document :}

Caciagli Mario, Baccetti Carlo, Négrier Emmanuel. Dernière étape avant Rome ? Les élections régionales italiennes du 16 avril 2000. In: Pôle Sud, n¹3, 2000. Qui gouverne les villes ? pp. 121-136;

doi : https://doi.org/10.3406/pole.2000.1091

https://www.persee.fr/doc/pole_1262-1676_2000_num_13_1_1091

Fichier pdf généré le 23/04/2018 


\begin{abstract}
The Italian regional elections, which occuredin april 16th 2000, took for the first time into account an electoral reform which consists in vote for the individual candidates to the présidence of Regions. This one was part of a more general trend toward the reinforcment of regional powers, which are often considered as rather weak in Italy. After an assessment of the detailed dynanmics which have marked these elections, the authors analyse, region by region, Berlusconi 's relatively modest victory. They consider that the weigh of changes affecting the system of coalitions is largely dominant in order to explain the contemporary landscape and to understand next political goals.
\end{abstract}

\title{
Résumé
}

Le scrutin régional italien du 16 avril 2000 prenait en compte, pour la première fois, la réforme électorale tendant à désigner personnellement les candidats à la présidence. Ce dispositif faisait partie d'un mouvement plus global destiné à renforcer le poids des régions, souvent considérées comme assez faibles en Italie. La personnalisation du pouvoir n'est pas très perceptible pour ce scrutin, marqué par un succès politique du centre-droit. Après avoir évalué en détail les dynamiques politiques qui ont marqué ces élections, les auteurs analysent, région par région, l'étendue, numériquement modeste, de la victoire de Berlusconi. Ils considèrent le poids des mutations dans le système d'alliance comme largement dominant pour expliquer la situation actuelle et les perspectives qui s'ouvrent pour 2001. 


\title{
Dernière étape avant Rome?
}

\section{Les élections régionales italiennes du 16 avril 2000}

\author{
Mario Caciagli et Carlo Baccetti \\ Département de Science Politique \\ Université de Florence
}

(Traduit de l'italien par Emmanuel Négrier)

\section{RésumélAbstract}

Le scrutin régional italien du 16 avril 2000 prenait en compte, pour la première fois, la réforme électorale tendant à désigner personnellement les candidats à la présidence. Ce dispositif faisait partie d'un mouvement plus global destiné à renforcer le poids des régions, souvent considérées comme assez faibles en Italie. La personnalisation du pouvoir n'est pas très perceptible pour ce scrutin, marqué par un succès politique du centre-droit. Après avoir évalué en détail les dynamiques politiques qui ont marqué ces élections, les auteurs analysent, région par région, l'étendue, numériquement modeste, de la victoire de Berlusconi. Ils considèrent le poids des mutations dans le système d'alliance comme largement dominant pour expliquer la situation actuelle et les perspectives qui s'ouvrent pour 2001.

The italian regional elections, which occured in april 16th 2000, took for the first time into account an electoral reform which consists in vote for the individual candidates to the presidence of Regions. This one was part of a more general trend toward the reinforcment of regional powers, which are often considered as rather weak in Italy. After an assessment of the detailed dynanmics which have marked these elections, the authors analyse, region by region, Berlusconi's relatively modest victory. They consider that the weigh of changes affecting the system of coalitions is largely dominant in order to explain the contemporary landscape and to understand next political goals.

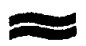

\section{Mots-clés/Keywords}

Élites régionales, Italie, loi électorale, partis politiques, personnalisation du pouvoir, Régions

Italy, electoral law, personalisation of power, political parties, regional elites, Regions 
Dans l'ensemble des 20 des régions italiennes, les élections ont lieu tous les cinq ans. Les régions à statut spécial (Val d'Aoste, 'Trentin-Haut Adige, Frioul-Venetie Julienne, Sardaigne et Sicile), nées à différentes dates entre 1946 et 1963, votent selon des échéances spécifiques. Les 15 régions à statut ordinaire ont en revanche toutes été instituées en 1968. Leurs premiers scrutins datent de 1970 , et les renouvellements se sont déroulés de cinq ans en cinq ans, régulièrement.

\section{Le vote régional dans le système politique italien}

Au cours de la Première République, les élections des régions à statut ordinaire, qui se tenaient en même temps que la plupart des élections municipales et provinciales, ne constituaient pas un rendez-vous politique de premier plan. Seules celles de 1975 apparurent-elles comme un moment crucial dans le système politique italien, par l'important succès alors remporté par le PCI, et par la formation de gouvernements de gauche (PCI, PSI et autres alliés minoritaires), au-delà des traditionnelles "régions rouges" (Emilie-Romagne, Toscane, Umbrie) en Piémont, Ligurie et Marches'.
Les élections régionales avaient peu de poids politique, parce que les institutions n'en avaient pas davantage. Nées en substance pour légiférer, coordonner et programmer les fonctions administratives des communes et provinces, les Régions à statut ordinaire étaient restées largement privées d'effectivité et de l'autonomie décisionnelle et, surtout, financière. L'État central avait évité de transférer ses compétences politiquement les plus significatives, conservant pour lui le pouvoir législatif dans tous les domaines importants, avec l'exception partielle de la santé ${ }^{2}$.

L'État italien demeurant centraliste, les carrières politiques des élus régionaux, et même de leurs présidents étaient restées presque étrangères au cursus honorum qui en revanche partait des postes municipaux ou provinciaux pour accéder au parlement national et, éventuellement, au gouvernement. L'élection régionale était l'aboutissement final de carrières politiques périphériques, à l'exception de quelques présidents devenus ministre ou député européen.

Bien des choses ont changé avec les élections de 1995, les premières de la longue transition entre les deux Républiques.

Tout d'abord, le mouvement "anticentraliste" a joué un rôle de premier plan dans la 
crise qui a secoué le système politique italien. Ce mouvement, s'il a trouvé un sujet politique, la Ligue Nord pour l'incarner efficacement, s'est étendu à de larges pans électoraux liés à tous les autres partis. Les critiques aux gouvernements romains successifs ont accru la demande d'une plus grande autonomie pour les niveaux sub-étatiques, par redistribution des ressources et de pouvoirs.

Les nombreuses mesures destinées à mettre en ouvre une décentralisation substantielle, orientées vers cette réforme fédéraliste de l'Etat italien que toutes les forces politiques déclaraient appeler de leurs voux, ont eu leur traduction dans les décrets de 1997, qui prirent le nom du ministre de la fonction publique qui les avait initiés et présentés, Franco Bassanini ${ }^{3}$. Une partie des décrets Bassanini a accru, ou prévu d'accroître en peu de temps le pouvoir décisionnel et financier des Régions.

En outre, dans le cadre de la réforme générale des systèmes électoraux italiens de tous niveaux (1993-19954) une nouvelle loi fut votée par le Parlement, en 1995, en vue des élections régionales. Elle établissait une prime majoritaire pour la coalition arrivée en tête. Les sièges supplémentaires, qui pouvaient varier au cas par cas selon les résultats, devaient garantir à la coalition victorieuse la majorité absolue et la stabilité gouvernementale. Le nouveau système, fidèle à l'esprit de toutes les réformes électorales de la transition italienne, devait stimuler la formation de coalitions inter-partisanes et favoriser ainsi la compétition bipolaire.

En fait, en 1995 pour la première fois dans l'histoire des élections régionales italiennes, se formèrent deux coalitions: centre-gauche et centre-droit, et ceci dans les 15 régions. Le centre-droit (il Polo), dont le leader était Silvio Berlusconi, était formé de Forza Italia, Alleanza Nazionale (AN) et du Centro Cris- tiano Democratico (CCD). Le centre-gauche était né d'une grande nouveauté, l'alliance des ex-communistes du Partito Democratico della Sinistra (PDS) avec les ex-démocrateschrétiens du Partito Popolare Italiano, une alliance dans laquelle entrèrent des groupes minoritaires comme les Verts et les socialistes de même que, dans bien des régions, Rifondazione comunista. Seuls restèrent en dehors des coalitions la Lega Nord, les radicaux de Pannella et les néo-fascistes de Rauti.

Ces élections enregistrèrent une victoire du centre-gauche, qui parvint à obtenir la majorité et à conquérir en conséquence le gouvernement dans 9 régions, contre 6 au centre-droit. La bipolarisation, déjà forte au cours de la campagne, fut accentuée par le caractère serré de certains résultats ${ }^{5}$. La victoire cimenta cette alliance entre PDS, PPI et autres, et donna vie à la coalition de l'Olivier, qui remporta les élections nationales suivantes. On peut donc dire que ces élections régionales préparaient le terrain du scrutin législatif suivant, en 1996, quand le même modèle de compétition (extériorité de la Lega Nord vis-à-vis des deux coalitions) rendait plus aisée une même victoire du centregauche.

\section{La signification politique des élections régionales de 2000}

L'image d'avant-première que les régionales de 1995 avaient eue pour les législatives de 1996 a chargé les élections de 2000 d'un sens particulier: celui d'un défi en vue des prochaines élections au Parlement, en 2001.

Les élections régionales de 2000 ont été en outre interprétées comme une évaluation par les électeurs de l'action des gouvernements de centre-gauche depuis 4 ans, et en particulier de celle du gouvernement D'Alema qui, 
comme on s'en souvient, avait succédé à Romano Prodi en octobre 1998.

Le jugement sur le gouvernement central, avec la transformation de la compétition régionale en compétition nationale, et le désintérêt pour l'évaluation des actions des gouvernements périphériques, a été en premier lieu porté par Berlusconi. Celui-ci a en fait imposé une campagne électorale étendue à tout le territoire national et il l'a conduite à la première personne du singulier, à l'aide de moyens pharaoniques (un navire de croisière avec lequel il a sillonné le pourtour italien), ainsi que, naturellement, toute la puissance de ses réseaux télévisuels. Berlusconi a attaqué constamment le "governo dei comunisti" et, en particulier, son président, le "communiste" D'Alema.

D'Alema a accepté ce type de défi. Luimême a affirmé, à partir de la phase centrale de la campagne électorale, que les résultats régionaux seraient un vote en faveur ou contre son gouvernement. Il faut dire que le choix stratégique de D'Alema a été en partie, sinon surtout fonction de raisons internes à la majorité de centre-gauche, dans la perspective du choix du meilleur candidat à la présidence du gouvernement. Si le vote régionale avait été favorable à celui-ci, D'Alema aurait eu en réalité de grandes chances d'être accepté par ses alliés comme candidat $\mathrm{n}^{\circ} 1$. Ceci semble avoir été en tout cas son calcul. C'est si vrai que, après s'être dépensé, personnellement et sur des thèmes de politique nationale, au cours de la campagne, il n'a pris que quelques heures pour démissionner et ouvrir la crise gouvernementale, dès qu'il fut clair que le centre-droit l'avait emporté. Avec cette décision, D'Alema a implicitement renoncé à se porter candidat au leadership du centre-gauche pour les prochaines élections législatives.

Quelques semaines plus tard se formait le gouvernement de Giuliano Amato, ex-diri- geant du défunt PSI, ex-président du Conseil en 1992 et ex-ministre du gouvernement D’Alema sans avoir été parrlementaire.

La claire reconnaissance de la défait de la part de D'Alema a renforcé le sens du triomphe du centre-droit, qui a, en toute cohérence, interprété le résultat des régionales comme l'étape ultime vers son "inévitable" victoire aux législatives. L'interprétation politique de la part du centre-droit se fondait (et se fonde encore) sur l'idée qu'il avait obtenu un succès retentissant, jugement partagé par les avis de bien des observateurs de différentes sensibilités politiques. Cette idée est soutenue par deux données incontestablement importantes. La première est que le centre-droit a conquis cette fois 8 régions sur 15 , qui représentent 32 millions d'habitants quand les 7 restantes n'en comptent que 16 millions. La seconde est que le centre-droit a conquis toutes les régions du nord, y compris la Ligurie, anciennement à gauche; c'est-à-dire les régions les plus peuplées, les plus développées et les plus riches du pays.

Cependant, d'autres données semblent redimensionner la victoire du centre-droit.

Tout d'abord, les coalitions qui se sont présentées n'étaient plus celles de 1995. La plus importante nouveauté réside dans le fait que le pôle s'est allié à la Lega Nord, avec les conséquences électorales et politiques sur lesquelles nous reviendrons.

En outre, et surtout, les données agrégées démontrent, comme nous le verrons dans les analyses qui suivent, que l'avantage du centredroit est limité. Une évaluation précise du jeu des alliances et la mesure des réels déplacements de votes incitent alors à une lecture des résultats différente de celle qui a prévalu.

Autrement dit, s'il reste incontestable que les élections régionales italiennes de 2000 ont conduit à une victoire politique du centredroit et une débâcle pour le centre-gauche, les 
données électorales ouvrent sur des réflexions plus mesurées. Comme les analyses chiffrées les plus autorisées l'ont noté, "la signification politique des élections ne correspond pas toujours aux résultats électoraux, de même que les convictions courantes sur les comportements électoraux ne trouvent souvent dans les chiffres les indices clairs qui les confirment" (Legnante, Corbetta, 2000).

Avant de passer à la présentation des données et à l'analyse, il est important de signaler l'ultime modification de la loi électorale régionale votée par le Parlement en décembre 1999.

\section{L'élection directe du Président de Région}

En vue des élections, le Parlement a modifié la loi électorale de 1995, au prix de la complexe procédure de révision de la Constitution. Il l'a fait avec la volonté convergente de la plupart des formations politiques, et pour satisfaire les exigences d'identification claire des responsabilités exécutives et de personnalisation de la politique. La loi de 1995 prévoyait déjà l'existence d'un chef de file des coalitions, mais ne pouvait imposer que celui-ci devint automatiquement président de région (même si cela advint de facto dans toutes les régions). Selon la nouvelle loi, les candidats à la présidence régionale, qui se présentent avec leurs propres colistiers (ceux de leur éventuelle prime majoritaire, dénommée "listino"), sont désignés à part par les électeurs qui ont ainsi à disposition deux votes pour exprimer un même choix; et si le vote se fait par liste bloquée, la désignation du président de son choix n'implique pas le vote en faveur de sa liste. En 2000 est donc advenue l'élection directe des quinze présidents de région, à l'instar des élections directes des maires, en 1993.
La nouvelle règle implique que, dans le cas où le président "élu par le peuple" n'aurait plus la majorité au Conseil, il soit automatique démis et que de nouvelles élections aient lieu. Cette règle a pour objectif d'empêcher que se forment des coalitions différentes de celles qui se sont présentées lors des élections. Elle vise donc à interdire ce que les italiens nomment le "ribaltone" : un ribaltone avait eu lieu lors de la précédente mandature en Campanie, lorsqu'un groupe d'ex-démocrateschrétiens a lâché la majorité de centre-droit pour former un nouveau gouvernement avec le centre-gauche, à la manière de ce qui avait provoqué la naissance du Cabinet D'Alema.

Avec la nouvelle situation, la chute d'un gouvernement régional sera donc très improbable. Difficile, mais pas formellement impossible, parce qu'un président "élu du peuple" peut aussi avoir la capacité de se chercher une nouvelle majorité.

Il reste vrai que l'élection directe du président de la Région répond aussi à l'exigence de renforcer les exécutifs et de conférer plus de prestige et plus de pouvoir à leurs dirigeants. Rappelons que les autres récentes lois sur l'autonomie régionale prévoient également un renforcement des pouvoirs des présidents. Cette élection directe répond incontestablement à la recherche générale des électeurs italiens d'une meilleure visibilité des candidats et d'une connaissance immédiate des vainqueurs. Les citoyens des quinze régions italiennes ont connu le soir même du 16 avril le nom de leur nouveau président de Région.

Il est cependant également vrai que les divers candidats, avec peu d'exception, n'avaient pas été les protagonistes d'une campagne dominée, comme on l'a vu, par l'omniprésence de Berlusconi et de son duel avec D'Alema. Plus, les présidents ont éprouvé de grandes difficultés à affirmer la centralité de leur rôle, lorsqu'il s'est agi de for- 
mer les équipes de gouvernement. Les "nouveaux" présidents, que la presse avait tôt fait de nommer "gouverneurs", devaient disposer de beaucoup de ressources d'autonomie décisionnelle, en faisant surtout valoir l'article 122 de la Constitution qui, une fois révisé, stipule que "le président nomme et révoque les membres de la giunta". Or plusieurs présidents ont été contraints à de longues et pénibles négociations avec tous les partis, grands et petits, de leurs coalitions pour parvenir à former leurs équipes et définir leurs missions.

En Toscane par exemple, le président Claudio Martini, membre des Démocrates de gauche (DS), parti depuis longtemps dominant dans la région, a dû accepter les pressions et requêtes de quelques dirigeants d'un parti petit mais important, le PPI, qui étaient en désaccord avec leurs propres camarades de parti. De même le président de la Région Lombardie, Roberto Formigoni, dirigeant de Forza Italia et président confirmé avec un score élevé de votes "populaires", a été contraint à bien des efforts pour satisfaire les prétentions d'Alleanza Nazionale et de la Lega Nord; et pour exercer une médiation entre ces deux forces politiques en conflit très dur. Pour former la giunta de la Région Veneto, les interventions des leaders nationaux ont été nécessaires pour obliger les parties à trouver un accord. Enfin, Antonio Bassolino (DS), maire très populaire (et populiste) de Naples et artisan du succès du centre-gauche en Campanie, a tenté d'exercer jusqu'au bout son pouvoir de nomination des membres du gouvernement, en choisissant plusieurs experts sans parti, mais a subi ainsi les attaques violentes des partis minoritaires de la coalition qui ont menacé d'un retour immédiat aux urnes.

Les choses ne sont pas allées mieux dans les autres régions: les intérêts des partis, des cou- rants et des groupes (jusqu’à ceux constitués d'un seul conseiller régional) ont partout surgi pour contraindre le "président élu du peuple" dans l'attribution des postes et fonctions.

Le fait est que le multipartisme existe et s'étend, même au niveau régional, et qu'il caractérise le système italien de la transition. D'autre part, la nouvelle loi électorale prévoit elle aussi des listes partisanes dans les circonscriptions électorales (qui correspondent aux provinces), et l'attribution des sièges à la proportionnelle. La compétition entre partis et, par le maintien du vote préférentiel, entre les candidats d'un même parti n'est pas atténuée par le fait que les listes provinciales soient liées à la liste régionale de la coalition, avec un symbole unique et le nom d'un candidat commun à la présidence.

\section{L'offre électorale: deux coalitions et un troisième acteur}

La compétition de l'an 2000 a vu s'affronter dans toutes les régions les deux formations qui, tout en étant en continuelle et notoire transformation, dominent la scène politique italienne depuis 1994.

Nous avons déjà signalé la grande nouveauté: l'adhésion au pôle de centre-droit de la Lega Nord, qui s'est partout présentée aux côtés des trois autres composantes du Pôle: Forza Italia, Alleanza Nazionale et le CCD. Dans certaines régions du centre et du sud, le petit parti néofasciste Fiamma Tricolore groupe dissident qui n'a pas accepté la métamorphose du MSI (Movimente Sociale Italiano) en Alleanza Nazionale - lui a apporté son soutien, en dépit des protestations et démentis.

La décision d'Umberto Bossi, toujours leader charismatique de la Lega Nord, d'abandonner, en vue de la campagne électorale, la 
situation d'isolement des cinq années précédentes et de rejoindre l'alliance avec le Pôle en a surpris plus d'un. Non seulement pour l'énième changement de stratégie (Bossi a déclaré renoncer pour le moment à la sécession et accepter un fédéralisme du possible), mais aussi pour les conflits très durs des cinq ans passés, souvent jalonnés d'insultes féroces entre la Lega et le Pôle après que la première eut causé la chute du gouvernement Berlusconi en décembre 1994, en sortant de la majorité.

Les difficultés de la Lega, organisationnelles et économiques plus que politiques, ont poussé Bossi à chercher à nouveau l'accord avec Berlusconi. Ce dernier ne pouvait pas ne pas être intéressé à reprendre la Lega sous son leadership, d'autant plus qu'elle y revenait dans une position clairement subalterne. Il est vrai en outre qu'une large part de l'électorat de la Lega voyait d'un bon cil le renouement de l'alliance, quand il n'était pas tout simplement prêt à voter Forza Italia.

Comme nous le verrons en chiffres, l'apport de la Lega n'a pas été déterminant pou le succès des candidats de centre-droit. Mais le mécanisme majoritaire des élections parlementaires rendra ce soutien utile pour assurer une véritable victoire à Berlusconi. C'est surtout dans ce sens que l'on peut considérer les élections régionales de 2000 comme une étape vers l'alternance gouvernementale nationale.

La coalition de centre-gauche était encore plus hétérogène et composite, au niveau régional comme il l'est au niveau national. DS, PPI, Verts, Rinovamento Italiano (le groupe du ministre des Affaires Etrangères, Lamberto Dini) et les Socialistes DémocratesChrétiens (SDI) avaient formé, en 1996, la coalition de l'Olivier, avec d'autres partis encore plus modestes. A ces partis ou groupes se sont associé ceux qui sont nés depuis deux ans: les Démocrates fondés par Romano Prodi en 1999, désormais dirigés par Arturo Parisi, et qui demeurent fidèle à L'Olivier; mais aussi ma anche deux petits partis radicalement hostiles à cette coalition, tout en faisant partie de la majorité gouvernementale à Rome: le Parti des Communistes Italiens (PdCI), né d'une scission du parti Rifondazione Comunista (PRC) en 1998, et l'Unione Democratica Europea (UDEur), constituée par des ex-démocrates-chrétiens passés de droite à gauche, dirigés par Clemente Mastella. Enfin, Rifondazione Comunista a adhéré aux coalitions de centre-gauche, avec la seule exception de la Toscane, où Rifondazione Comunista a présenté son propre candidat à la présidence, avec son "listino".

Les accords avec Rifondazione dans presque toutes les régions sont un indice de disponibilité réciproque pour une entente électorale lors des législatives, même si la définition de la forme et des contenus supposera des négociations délicates.

Au-delà de ces deux coalitions, il faut rappeler que, outre quelques listes mineures présentes dans quelques régions, un troisième acteur était par contre présent dans toute l'Italie: la liste des radicaux qui, cette fois, s'était donné pour nom celui d'Emma Bonino, membre de la Commission Européenne. La Liste Bonino avait recueilli un score notable aux européennes de 1999 , avec $8,7 \%{ }^{6}$ des voix, et était ainsi considérée comme une troisième voie, dotée d'un poids électoral certain grâce à un probable exploit électoral. Pour cette raison, les dirigeants des deux Pôles avaient tenté d'ouvrir un dialogue avec elle.

\section{Les résultats}

La forte chute des scores de la Liste Bonino est peut-être la surprise majeure du vote du 16 avril. A dix mois de distance des élections 


\section{Tobleav 1 - Electlone reolonales 2000}

Total dos volx on taveur dos libtes dent Ponsemble des collbges provinclaux

\section{Valeur Absolue}

Centre-droit

Liste Bonino

Centre-gauche

Autres

Total
13238210

558791

11362285

212817

25372103
Pourcentages

$52,2 \%$

$2,2 \%$

$44,8 \%$

$0,8 \%$

$100,0 \%$

Source: G. Legnante, P. Corbetta, "Cambiamento politico e stabilita elettorale", in il Mulino, n. 3, 2000.

Tablenu 2 - Elections roglonales 2000

Total des votes pour les candidats a la presidence dans Pensemble des réglons

Valeur Absolue

14312762

714942

12866685

345165

28237554
Pourcentages

$50,7 \%$

$2,5 \%$

$45,6 \%$

$1,2 \%$

$100,0 \%$

Source: G. Legnante, P. Corbetta, "Cambiamento politico e stabilita elettorale", in il Mulino, n. 3, 2000. Elaboration des auteurs.

européennes, elle est descendue à un modeste $2,2 \%$ du total national. La faible participation, la notoriété de la commissaire européenne, et le soutien massif que lui avait consenti Berlusconi sur ses réseaux télévisuels avaient été les facteurs principaux de son incroyable succès en 1999. L'absence de tels facteurs en 2000 explique encore plus facilement un tel résultat. Le pourcentage de 2,2\% correspond à un total d'un peu plus de 500000 voix au total, comme on peut le voir sur le tableau $n^{\circ} 1$.

Sur ce même tableau, on peut voir aussi les chiffres absolus et en pourcentage qui montrent la nette victoire du centre-droit.

Les scores obtenus par les listes des deux coalitions, comme ceux obtenus par la Liste
Bonino, sont restés inférieurs à ceux recueillis par leurs respectifs candidats à la présidence régionale. On peut comparer les tableaux 1 et 2 .

Cette différence de chiffre pourrait signifier qu'une partie des électeurs a voté seulement pour les candidats, les préférant aux listes ou coalitions. Mais la différence entre les totaux (qui pourrait être due en partie à des erreurs par omission dans les choix individuels) est, comme on le voit, encore minime par rapport aux attentes diffuses d'une personnalisation accentuée de la compétition. Les enquêtes dans les régions pendant la campagne ont d'ailleurs confirmé que les candidats à la présidence sont encore peu connus, bien moins en tout cas que les 
leaders nationaux. Les analyses approfondies des votes agrégés confirment que lors de ces régionales, il n'y a pas eu de personnalisation croissante: les votes en faveur des listes régionales et pour les candidats à la présidence ont été plus nombreux dans le Nord, tandis que prévalaient, au sud, les votes en faveur des partis. C'est peut-être ici le produit du "réseau de mobilisation des candidats internes aux listes pour la capture des votes préférentiels" (Agosta, 2000).

Les pourcentages du tableau $n^{\circ} 2$ indiquent toutefois que les candidats du centre-gauche ont recueilli un score légèrement meilleur que leurs listes; mais il n'en est pas de même pour les candidats de centre-droit. Quelques excellentes candidatures individuelles de centregauche (comme celles de Massimo Cacciari dans le Veneto, d'Antonio Bassolino en Campanie) y ont contribué. Mais même dans ces deux cas, comme dans tous les autres, l'"effet candidat" a été inférieur aux prévisions. Nonobstant le nouveau mode d'élection directe du président, les électeurs ont continué à voter pour les listes et partis.

Afin de confronter ces résultats à ceux des précédentes élections régionales, tout en restant prudent au vu des modifications du système électoral et des alliances, on peut comparer les scores nationaux obtenus par les présidents en 2000 avec les pourcentages obtenus par les listes majoritaires en 1995. Le centre-gauche, qui a obtenu $45,6 \%$ des voix en 2000 (tableau $n^{\circ} 2$ ), avait atteint $41,2 \%$ en 1995 , auxquels il faut ajouter les $5,6 \%$ de Rifondazione Comunista: le résultat se traduit par la perte de 2,2\% des voix. Le centredroit, qui a atteint $50,7 \%$ en 2000 , avait obtenu $41,6 \%$ en 1995 , auxquels on doit ajouter les 6,6\% de la Lega Nord : ceci représente un gain de $2,5 \%$.

Ce sont surtout ces deux chiffres (perte de 2,2 points d'un côté et gain de 2; 5 de l'autre) qui donnent la mesure exacte du résultat de ces élections. On peut convenir que ce sont des changements modestes qui, comme on l'a indiqué, n'incitent ni au triomphe ni à la déconfiture.

On peut plutôt faire le décompte des votes à l'intérieur des coalitions elles-mêmes. Sur la base provinciale, proportionnelle, il est possible d'obtenir une totalisation nationale qui donne une approximation relative de l'influence électorale des différentes forces politiques. On parle d'approximation relative parce que les partis isolés ne se sont pas présentés dans toutes les provinces, ou parce qu'ils se sont présentés parfois sur des listes communes.

Les conclusions fournies par les services électoraux du Ministère de l'Intérieur ${ }^{7}$ indiquent ainsi un grand succès pour Forza Italia, qui aurait réuni dans l'ensemble des régions $25,4 \%$ des voix, s'affirmant incontestablement comme le premier parti.

Pour les autres partis, il est plus opportun de se fier à l'estimation d'une étude statistique qui a pris en compte le fait que ceux-ci ne se sont pas présentés dans toutes les régions avec leurs propres sigles. Selon cette expertise, les DS sont confirmés comme second parti mais, avec 19,2 \%, sont loin de Forza Italia. Alleanza Nazionale se voit attribuer $13,1 \%$, au PPI 5,7 \%, au CCD 3,8 \%, à UDEur, aux socialistes et aux Verts $2,5 \%$ chacun. Tous ces partis, si ces estimations sont fiables, apparaissent ainsi stables par rapport aux élections antérieures. Les seules formations qui subissent des pertes sont les radicaux de Bonino et les Démocrates de Prodi et Parisi, qui sont passés de 7,7\% aux européennes à 4,7\%. La Lega Nord est en baisse constante, avec 4,9\% dans l'ensemble des régions où elle se présentait, tout comme Rifondazione comunista, qui a également obtenu 4,9\% sur l'ensemble du territoire national ${ }^{8}$. 


\begin{tabular}{|c|c|c|c|}
\hline \multicolumn{2}{|c|}{ Centre-droit } & \multicolumn{2}{|c|}{ Centre-gauche } \\
\hline 1995 & 2000 & 1995 & 2000 \\
\hline Piemonte & Piemonte & Liguria & \\
\hline Lombardia & Lombardia & Emilia Romagna & Emilia Romagna \\
\hline Veneto & Veneto & Umbria & Umbria \\
\hline - & Liguria & Toscana & Toscana \\
\hline & Lazio & Marche & Marche \\
\hline Abruzzo & Abruzzo & Lazio & \\
\hline Campania & & & Campania \\
\hline Puglia & Puglia & Molise & Molise \\
\hline & Calabria & Basilicata & Basilicata \\
\hline & & Calabria & \\
\hline
\end{tabular}

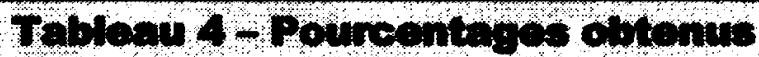
per los Prodictute olus dens tos 15 rodons

Région Centre-droit

Plemonte Enzo Ghigo (FI)

Lombardia Roberto Formigoni (FI)

Liguria

Sandro Biasotti (FI)

Veneto

Giancarlo Galan (FI)

Lazio

Francesco Storace (AN)

Abruzzo

Giovanni Pace (AN)

Puglia

Raffaele Fitto (FI)

Calabria

Giuseppe Chiaravallotti (FI)

Région Centre-gauche

$\%$

Emilia-

Romania Vasco Errani (DS)

Toscana Claudio Martini (DS)

56,5

Marche

Vito D'Ambrosio (DS)

49,2

49,9

Umbria Maria P. Lorenzetti (DS)

Molise Giovanni Di Stasi (DS)

Campania Antonio Bassolino (DS)

Basilicata Filippo Bubbico (PPI)

54,9

51,3

49,3

\section{Le vote région par région}

Nous avons annoncé que d'autres aspects, politiquement plus importants, des résultats de ces élections donnent raison au centre51,8 droit. Examinons, dans le tableau ci-dessus, 62,4 les régions conquises ou perdues par l'une ou 50,8 l'autre des coalitions.

Le centre-droit a ainsi perdu une région et en a conquis trois. C'est l'inverse pour le centre-gauche, qui peut revendiquer dans le Sud la Campania, seconde région italienne en nombre d'habitants, avec Naples pour capitale, et se consoler de la perte d'une région pauvre et aussi petite que la Calabre.

Mais le centre-droit, même si la perte de la Campania est un coup dur, peut se réjouir de bien plus: la conquête de la Ligurie et du Latium, même si cela fut obtenu de justesse.

La Ligurie a souvent fait partie, lors des cinquante dernières années, des régions rouges et sa capitale, Gènes, a été pendant un siècle l'une des places fortes du mouvement ouvrier italien. Plus, maintenant, le centre-droit contrôle désormais tout le nord qui au poids économique et démographique ajoute les inquiétudes autonomistes. Les 
quatre présidents de régions septentrionales, à peine élus, ont commencé à faire entendre leurs revendications contre le gouvernement de Rome.

Significative en termes symboliques plus encore qu'en termes de pouvoir est la conquête par le centre-droit du Latium, la région de la capitale, Rome. Ajoutons encore au symbole en indiquant que le candidat de cette coalition était un dirigeant d'Alleanza Nazionale, Francesco Storace, connu pour son passé d'agitateur néo-fasciste et pour son appartenance à une "droite sociale", à l'idéologie anti-égalitaire et répressive ; caractéristiques qui, selon bien des observateurs, n'auraient pas pu lui permettre d'avoir le soutien de bien des électeurs modérés.

Le tableau 4 mentionne les noms des présidents élus dans les 15 régions, avec les pourcentages obtenus et les partis auxquels ils adhèrent.

Certaines de ces données régionales méritent un commentaire.

Le pourcentage de $62,4 \%$ atteint par Formigoni en Lombardie est considérable. L'alliance du Pôle avec la Lega Norda a donné au centre-droit son meilleur résultat dans la plus grande et la plus européenne région italienne. Affilié à Forza Italia à la tête d'un petit groupe d'ex-démocrates-chrétiens liés à un mouvement clérical et intégriste, Formigoni a pratiquement étrillé son adversaire du centregauche, Mino Martinazzoli, l'ultime secrétaire de la Démocratie Chrétienne, qui contribua à la naissance du Partito Populare.

Laffirmation des candidats de centre-droit dans le Veneto a été positive, grâce à une Lega en crise mais toujours forte, de même que dans les Pouilles, où l'apport décisif est venu d'Alleanza Nazionale. On ne peut en dire autant dans les autres régions, là où la compétition était plus ouverte (Ligurie, Latium).
Les candidats de centre-gauche l'ont emporté, comme c'était aisément prévisible, dans les régions rouges: largement en Emilie-Romagne et Ombrie, moins facilement en Toscane (mais ici, Rifondazione Comunista ne faisait pas partie de la coalition) et dans les Marches. Dans ces quatre régions, les DS conservent un rôle prédominant, même si la nouvelle formation n'a certainement plus la capacité organisationnelle et l'ancrage social du PCI.

Le score élevé recueilli par Filippo Bubbico, candidat du centre-gauche en Basilicate, n'a, en revanche, pas été dû au PDS mais à la présence d'un Parti Populaire fort, qui a capté la plus grande partie de l'héritage démocratechrétien, qui avait bien gouverné cette région à la fois petite et en fort développement. La conquête de la Campanie, très importante, a été d'une ampleur moins forte que prévu, avec $54,2 \%$ obtenus par Bassolino.

Le tableau 4 rassemble les pourcentages atteints par les listes régionales (qui se rapportent, comme on l'a vu en examinant le système électoral, à l'élection des têtes de listes en 1995, et à celle des présidents pour 2000) lors des deux consultations. Dans les données de 1995, les données du Pôle et du centre-gauche sont respectivement séparées de celles de la Lega Nord, et de Rifondazione Comunista.

Comme on le voit, le centre-droit est en croissance dans presque toutes les régions. Au Nord, le Veneto fait exception, sans doute à cause des divisions internes et de l'affaiblissement de la Lega. En outre, au Piémont et en Lombardie, le Pôle aurait assurément vaincu sans l'apport de la Lega. Enfin, c'est en Ligurie que le centre-droit a enregistré sa plus forte croissance $(+6,2 \%)$.

Le centre-droit s'est également renforcé dans les régions rouges. Notons sa croissance, sans la Lega, dans les Marches $(+5,3 \%)$, les Pouilles (où il était déjà fort) et en Calabre. 


\begin{tabular}{|c|c|c|c|c|c|c|}
\hline Regione & $\begin{array}{c}\text { Polo + LN } \\
2000\end{array}$ & $\begin{array}{c}\text { Polo + LN } \\
1995\end{array}$ & $\begin{array}{l}\text { Polo + LN } \\
2000 / 1995\end{array}$ & $\begin{array}{c}C s \\
2000\end{array}$ & $\begin{array}{c}\text { CS + PRC } \\
1995\end{array}$ & $\begin{array}{c}\text { CS + PRC } \\
2000 / 1995\end{array}$ \\
\hline Piemonte & 51,8 & $\begin{array}{c}39,7+11,1 \\
(50,8)\end{array}$ & $+1,0$ & 39,5 & $\begin{array}{c}35,2+9,3 \\
(44,5)\end{array}$ & $-5,0$ \\
\hline Lombarclia & 62,4 & $\begin{array}{c}41,6+17,7 \\
(59,3)\end{array}$ & $+3,1$ & 31,5 & $\begin{array}{c}27,6+8,2 \\
(35,8)\end{array}$ & $-4,3$ \\
\hline Veneto & 54,9 & $\begin{array}{c}38,2+17,5 \\
(55,7)\end{array}$ & $-0,8$ & 38,2 & $\begin{array}{c}32,3+6,9 \\
(39,2)\end{array}$ & -1 \\
\hline Liguria & 50,8 & $\begin{array}{c}38,0+6,6 \\
(44,6)\end{array}$ & $+6,2$ & 46,0 & $\begin{array}{c}42,4+8,6 \\
(51)\end{array}$ & $-5,0$ \\
\hline Emilia-R. & 40,3 & $\begin{array}{c}32,0+3,8 \\
(35,8)\end{array}$ & $+4,5$ & 56,5 & $\begin{array}{c}53,8+8,8 \\
(62,6)\end{array}$ & $-6,1$ \\
\hline Romania & 40,0 & 36,1 & $+3,9$ & $\begin{array}{c}49,2+7,7^{*} \\
(56,9)\end{array}$ & $\begin{array}{c}50,1+12,4^{*} \\
(62,5)\end{array}$ & $-5,6$ \\
\hline Umbria & 39,2 & 39,0 & $+0,2$ & 56,4 & 59,9 & $-3,5$ \\
\hline Marche & 44,2 & 38,9 & $+5,3$ & 49,9 & 51,6 & $-1,7$ \\
\hline Lazio & 51,3 & 48,0 & $+3,3$ & 46,0 & 48,1 & $-2,1$ \\
\hline Abruzeo & 49,3 & 47,2 & $+2,1$ & 48,8 & 48,2 & $+0,6$ \\
\hline Molise & 48,7 & 49,5 & $-0,8$ & 49,0 & 50,5 & $-1,5$ \\
\hline Campania & 44,7 & 47,9 & $-3,2$ & 54,2 & 39,3 & $+14,9$ \\
\hline Puglia & 54,0 & 49,8 & $+4,2$ & 43,4 & 45,8 & $-2,4$ \\
\hline Basilicata & 35,1 & 36,6 & $-1,5$ & 63,1 & 54,8 & $+8,3$ \\
\hline Calabria & 49,8 & 44,2 & $+5,6$ & 48,7 & $\begin{array}{c}38,0+9,6 \\
(47,6)\end{array}$ & $+1,1$ \\
\hline
\end{tabular}


En Campanie, il a subi sa perte la plus grave: le passage dans l'autre camp du petit parti de l'UDEur et la candidature de Bassolino ont condamné le centre-droit à son unique réelle défaite.

La seule victoire du centre-gauche, à travers le passage de $39,3 \%$ en 1995 à $54,2 \%$ en 2000 , est due aux raisons invoquées plus haut. La croissance en Basilicate (de 54,8\% à $63,1 \%$ ), due au rôle des popolari, est l'autre succès important: mais le Basilicate est une région très petite.

Le centre-gauche et, en son sein, les DS sont préoccupés par leur recul dans les régions rouges, même si l'alternance de centre-droit semble n'être, au moins en termes numériques, qu'une hypothèse lointaine. Le plus grave est sans doute réside dans les importantes pertes dans les régions du Nord, où la domination du centre-droit en fait désormais une rampe de lancement pour un triomphe lors des prochaines élections législatives.

\section{Les élections dans deux régions: Veneto et Toscane}

Il y avait plusieurs bonnes raisons de porter une attention particulière aux résultats venètes. C'est la région où, à la fin des années 1980, s'étaient enracinés avec le plus de force sur le territoire les premiers mouvements antipartitocratiques et anti-centralistes; la région, donc, qui avait plus que d'autres contribué à l'écroulement de la DC.

Après les élections de 1995, une majorité confortable s'était établie au gouvernement venète, avec à sa tête un dirigeant de Forza Italia, qui se représentait en 2000. La Legal'avait cette fois rejointe, comme dans tout le Nord, quoique les divergences entre les deux formations y aient été particulièrement après et durables.
Le premier élément d'incertitude était justement de savoir si les électorats respectifs de la Lega et de Forza Italia, plutôt proches idéologiquement, auraient facilement oublié les années de reproches et d'accusations entre leurs dirigeants, pour faire prévaloir la nouvelle option d'une coalition.

Cette incertitude était renforcée par le fait que le pôle adverse présentait Massimo Cacciari, le maire de Venise, comme candidat à la présidence. Par sa notoriété et son image publique, il était considéré comme particulièrement "capable d'intercepter des courants d'opinion transversaux, dépassant les clivages institués" (Diamanti, 2000).

La candidature de Cacciari semblait également un test plus probant pour vérifier l'incidence de la personnalisation, le poids qu'aurait le facteur individuel dans la compétition pou la présidence. Pour tirer le meilleur profit de son équation personnelle, le maire de Venise avait formé une liste à son nom, la Lista Cacciari, dans laquelle s'inséraient les groupes les plus importants du centre: les Démocrates et le PPI.

La réponse des urnes a balayé ces incertitudes. la Lega et le Pôle unis ont quasiment confirmé le résultat obtenu en 1995, quand ils s'étaient présentés séparément; le centregauche et Rifondazione Comunista n'ont obtenu qu'un point de mieux. Cette continuité de comportement d'un électorat conservateur a eu un effet amplificateur sur la distance entre les deux candidats à la présidence; l'écart, qui était de 6 points en 1995 , est en 2000 de 16; le centre-droit obtenant 60,2\% contre $33,8 \%$ pour le centre-gauche (un écart de $26 \%$ !). La candidature de Cacciari a montré, même partiellement, l'effet de la personnalisation: il a réuni plus de suffrages $(+4,4 \%)$ que les listes qui le soutenaient. Le candidat de centre-droit a, lui, été en retrait de 5,3\% visà-vis du score de sa liste. Cacciari a fait croître 
le centre-gauche par rapport à sa consistance effective dans le Veneto, mais "n'a pas empêché l'écart entre les deux coalitions de rester très ample ${ }^{9 \prime}$.

Une autre région méritait un bref commentaire: la Toscane. Ici, les raisons sont très différentes du Veneto, comme le sont les couleurs politiques. La Toscane, toujours gouvernée par le PCI puis par le PDS, a été le seul cas où il n'y a pas eu d'accord électoral entre le centre-gauche et Rifondazione Comunista, très forte dans la région.

La réaction de l'électorat de gauche à cet accord manqué était donc attendue, avec notamment la crainte d'une croissance de l'abstentionnisme dans ce camp. Le centredroit comptait dessus pour ouvrir la première brèche (en 1995, la coalition de l'Olivier et le PRC avaient conquis tous les sièges majoritaires sauf un $\left.{ }^{10}\right)$. Le Pôle, ici aussi allié à la Lega (quoique cette dernière n'ait jamais eu beaucoup d'influence en Toscane), espérait affaiblir la gauche, en comptant aussi sur le fait que le président sortant, Vannino Chiti, homme politique reconnu et doté d'une équation personnelle certaine, avait renoncé à se représenter. Cependant, le candidat retenu par le Pôle ne semblait pas à beaucoup le plus adapté pour lancer l'offensive contre la majorité sortante. Altero Matteoli est un homme politique de l'Alleanza Nazionale, assez connu (ex-ministre de l'environnement du gouvernement Berlusconi en 1994), mais clairement situé à l'extrême-droite de la coalition. Sa trajectoire de néo-fasciste ne semblait pas des plus recommandées pour déplacer vers le centre-droit les suffrages d'un électorat comme celui de Toscane qui a toujours donné la preuve, pour des raisons historiques précises, d'un sentiment anti-fasciste profond et diffus.

Une fois ouvertes les urnes, on a vu en effet que la tradition politique régionale en sortait confirmée, mais quelques éléments d'inquiétude pour la majorité étaient introduits; de sorte que le centre-droit pouvait peut-être commencer à penser à la Toscane comme une région éventuellement prenable. Le candidat de centre-gauche, l'ex-assesseur régional Claudio Martini, sortait vainqueur avec un score qui restait, même de peu, en dessous de la majorité absolue des suffrages exprimés (49,3\%), à un point de l'influence propre des listes de gauche $(50,3 \%)$; une manière de démontrer comment, dans ce cas, la candidature personnelle en soi n'a produit aucune valeur ajoutée. Le candidat comme la coalition de centre-gauche ont perdu des points (respectivement $0,8 \%$ et $2,5 \%$ ) par rapport à 1995. Le jeune candidat du PRC et la liste partisane ont subi également un revers: un véritable écroulement par rapport à 1995 (de $11,1 \%$ à $6,7 \%$ pour la liste; de $12,4 \%$ à $7,7 \%$ pour la candidature à la présidence). C'est donc l'ensemble de la gauche qui est en recul.

Détrompant bien des prévisions, Matteoli a atteint $40 \%$ (quasiment le même score que la liste proportionnelle: $40,5 \%$ ), un résultat de quatre points meilleurs que celui du Pôle cinq années auparavant (Paolo Del Debbio, leader national de Forza Italia, plus "présentable" que Matteoli aux yeux d'un électorat modéré, mais moins connu), tandis qu'entre listes, la progression est de près de 6 points. En somme, le parcours néo-fasciste du candidat n'a ni ralenti ni freiné le regain du centre-droit, qui a substantiellement réduit l'écart vis-à-vis de son adversaire en se portant à "seulement" 10 points, même s'il faut tenir compte des suffrages pour le moment "gelés" du PRC.

La participation électorale a certainement influé sur le vote toscan et sur l'affaiblissement de la gauche. Le taux d'abstention a progressé de $10 \%(25,4 \%$ contre $14,9 \%$ en 
1995) et s'est désormais aligné avec la moyenne nationale $(27,4 \%)$, inversant une tradition qui voyait dans la participation électorale l'une des majeures "vertus civiques" des régions rouges".

\section{L'électorat stable d'un sys- tème en mutation}

Après ses succès aux élections municipales et européennes de 1999, et après l'échec du référendum pour l'abolition de la proportionnelle dans les élections législatives, que Berlusconi considérait comme sa troisième victoire, ce dernier a pu exulter pour un quatrième succès consécutif. Celui-ci semble surtout projeter Forza Italia vers la victoire définitive aux législatives et le retour de son leader à la direction du gouvernement. Le système politique italien connaîtrait pour la seconde fois en cinq ans l'alternance gouvernementale de deux coalitions opposées, après 45 ans d'immobilisme dominé par le même parti : la DC.

Mais si le centre-droit succède au pouvoir au centre-gauche, le mécanisme de l'alternance sera actionné, y compris cette fois, plus par les élites politiques que par les électeurs. L'électorat italien, qui a toujours été très stable, l'a démontré une nouvelle fois en ces années de transition. Les déplacements de voix ont été assez modestes et ont été enregistrés dans les partis d'une même coalition, et non entre coalitions, comme cela $s^{\prime}$ est à nouveau produit pour les régionales ${ }^{12}$.

Dans toutes les élections qui se sont déroulées de 1994 à 2000, le centre-gauche a toujours oscillé autour de $44 \%$, et le Pôle autour de $46 \%$. Les victoires de la coalition progressiste en 1995 et 1996, comme nous l'avons rappelé dans ces pages, ont été dues non à une réelle majorité d'opinion dans le pays, mais à un mécanisme électoral qui a pénalisé les forces conservatrices quand celles-ci se sont présentées divisées.

C'est la Lega Nord qui a décidé de l'issue de ces élections, alliée à Berlusconi en 1994 et devenant son adversaire. La Lega ne s'est pas alliée avec le centre-gauche, mais sa candidature autonome a permis à celui-ci de l'emporter aux régionales de 1995 et aux législatives de 1996. Quand la même Lega Nord s'en est retournée vers le Pôle en 2000, le centre-droit a de nouveau vaincu; et, s'il demeure uni, il l'emportera presque sûrement aux prochaines législatives.

Si le centre-droit gagne ces élections, ce sera l'écho d'un trait historique de fond de l'électorat italien. Dans sa grande stabilité, il avait connu, pour toute la première République, une seule et constante tendance politique conservatrice. Cette tendance n'a pas disparu au cours de la transition. Lors des élections régionales de 2000 , elle s'est illustrée en toute clarté. 


\section{Notes}

1. Sur ces élections, on peut encore se référer à C. Ghini (1976). Sur ce tournant et le rôle des élections régionales pendant la Première République, $f f$. De Mucci, Massari et Parisi (1987).

2. Sur les limites qui ont marqué depuis l'origine le rôle des régions en Italie, cf. Dente (1989) et Rotelli (1991).

3. Pour une discussion approfondie de la nouveauté introduite par les décrets Bassanini (Lois 59/1997 et 127/1997), voir le fascicule monographique de la revue le Regioni, $n^{\circ}$ 5, 1997.

4. Il faut se rappeler que, après la crise de la Première République, les reformes électorales ont été considérées par la classe politique, mais aussi par une partie de l'opinion, comme le premier et nécessaire pas vers le changement du système politique et son fonctionnement.

5. Sur les élections régionales de 1995, of. les commentaires et interprétations de Di Virgilio $(1995 ; 1996)$ et D'Alimonte (1995).

6. Pour un commentaire des résultats de ces élections, $f f$. Daniels (2000).

7. Ministero dell'Interno, Direzione centrale per i Servizi elettorali, 16 aprile 2000. Elezioni regionali Roma, 2000.

8. Toutes les estimations et leurs commentaires dans P. Scaramozzino, "Regionali: Quercia e non-DS in equilibrio", Il Sole-24 Ore, 23 avril 2000.

9. "Elezioni regionali: il comportamento di voto nel Veneto", numéro spécial de Nord Est, n 4, avril 2000.

10. Sur la dynamique électorale de Toscana, $f f$. Caciglia, Baccetti (1998).

11. Une analyse complète, soignée et approfondie des élections régionales en Toscane se trouve dans Floridia (2000).

12. La seule vraie nouveauté des années 1990 dans le comportement électoral italien a été la montée de l'abstentionnisme... Lors des élections 2000 , la participation électorale est tombé a $72,6 \%$, contre $81,4 \%$ lors des régionales de 1995.

\section{Références}

Agosta A., "Le incerte geometrie della politica debole", Il Sole/24 ore, 24 juin 2000.

Caciagli M., Baccetti C., "Dynamiques électorales et forces politiques en Toscane", Pôle Sud̆ n 8, 1998.

D'Alimonte R. "La transizione italiana: il voto regionale del 23 aprile", Rivista italiana di scienza politica, $\mathrm{n}^{\circ} 3$, 1995.

Daniels P., "Le elezioni del Parlamento europeo del 1999", in M. Gilbert, G. Pasquino (dir.), Politica in Italia Edizione 2000, il Mulino, Bologna, 2000.

De Mucci R., Massari O., Parisi A., in M. Caciagli, P. Corbetta (dir.), Elezioni regionali e sistema politico nazionale, Bologna, il Mulino, 1987.

Dente B., "Il governo locale", in G. Freddi (dir.), Scienza dell'amministrazione e politiche pubbliche, Roma, La Nuova Italia Scientifica, 1989.

Di Virgilio A., "Le elezioni in Italia", Quaderni dell'Osservatorio elettorale, $n^{\circ} 34,1995$.

Di Virgilio A., "Le elezioni regionali e amministrative: bipolarizzazione con riserva", in M. Caciagli, D. Kertzer (dir.), Politica in Italia - Edizione 96, Bologna, Il Mulino, 1996.

Diamanti I., "Veneto: la personalizzazione imperfetta", Il Mulino, n 3, 2000.

Floridia A., "La Toscana è ancora una regione rossa? Note e riflessioni sulle elezioni regionali del 16 aprile 2000", Quaderni dell'Osservatorio elettorale, $\mathrm{n}^{\circ} 43$, juin 2000.

Ghini C., Il terremoto del 15 giugno, Roma, Editori Riuniti, 1976

Legnante G., Corbetta P., “Cambiamento politico e stabilità elettorale”, Il Mulino, n 3, 2000.

Rotelli E., Dalla parte delle autonomie, Roma, Collana Editoriale ANCI, 1991. 